\section{Zum Problem elektronischer Eigenleitung von Halbleitern}

\author{
Von K. W. B ö e r
}

II. Physikalisches Institut der Humboldt-Universität Berlin

(Z. Naturforschg. 10 a, 898-899 [1955] ; eingeg. am 22. September 1955)

Die elektronische Leitfähigkeit von Halbleitern bei höheren Temperaturen nimmt im allgemeinen exponentiell mit der Temperatur zu. Die Größe der Leitfähigkeit sowie der Exponent ihres Anstieges hängen dabei im Bereich mittlerer Temperaturen empfindlich von der Realstruktur des Halbleiters, also z. B. vom Verunreinigungsgrad ab. Man spricht daher gewöhnlich in diesem Bereich von einer elektronischen Fremdleitung. Die Fermi-Grenze liegt nicht in der Mitte der verbotenen Zone, sondern dicht an einem Bandrand (am Leitungsband für Überschußleiter und am Valenzband für Defektleiter); ihre Lage wird wesentlich durch Donatoren oder Akzeptoren bestimmt.

Im Bereich höherer Temperaturen verschwindet im allgemeinen der Einfluß von Verunrẹinigungen oder der Vorbehandlung auf die Leitfähigkeit. Der Exponent ihres Anstieges wird größer und stimmt in vielen Fällen recht gut mit dem Wert überein, den man aus der aus optischen Absorptionsuntersuchungen gefundenen Breite der verbotenen Zone errechnet. Hier wird dann gewöhnlich von einer elektronischen Eigenleitung gesprochen; die Fermi-Grenze sollte demnach jetzt in der Mitte der verbotenen Zone liegen. Damit kann aus dem gemessenen Leitfähigkeitsanstieg direkt die Stromträgerkonzentration (Elektronen oder Defektelektronen) bei vorgegebener Temperatur berechnet werden.

$$
n=2\left(\frac{\sqrt{m_{\mathrm{p}} m_{\mathrm{n}}} k T}{2 \pi \hbar^{2}}\right)^{3 / 2} \cdot e^{-\Delta E / 2 k T .}
$$

Dann ist aus der gemessenen Leitfähigkeit selbst unter Benutzung von

$$
\sigma=2 \text { en } b
$$

die Stromträgerbeweglichkeit * angebbar, wenn für $n$ die aus dem Leitfähigkeitsanstieg berechnete Stromträgerkonzentration eingesetzt wird.

Diese Bestimmung der Stromträgerbeweglichkeit aus der Leitfähigkeit im Gebiet hoher Temperaturen ist an einer großen Zahl verschiedener Halbleiter ausgeführt worden und ergibt im allgemeinen Werte, die u. U. um viele Größenordnungen kleiner sind als die nach den üblichen Methoden bestimmten Beweglichkeiten (z. B. durch Hall-Effekt). Es ist sehr unwahrscheinlich, daß die meist nur sehr ungenau bekannten effektiven Massen $m_{\mathrm{n}}$ und $m_{\mathrm{p}}$ diese beträchtliche Diskrepanz, die beim CdS und Se mehr als 5 Größenordnungen ausmacht, allein bedingen.

Weitere unsichere Faktoren, die für einen derartig großen Effekt verantwortlich gemacht werden können, gehen jedoch in diese einfache Überlegung nicht ein, so daß zu einer Klärung die Voraussetzungen zur Anwendung der ersten Gleichung näher zu untersuchen sind. Es ist danach zu entscheiden, ob in dem zur Diskussion stehenden Temperaturbereich tat-

* Im Rahmen der hier durchgeführten Betrachtungen soll die Elektronenbeweglichkeit der Defektelektronenbeweglichkeit gleichgesetzt werden. sächlich bereits eine echte Eigenleitung auftritt, oder ob sie nicht durch andere Effekte lediglich vorgetäuscht wird.

Dazu wurden in diesem Temperaturgebiet nichtstationäre Untersuchungen angestellt. Untersuchungssubstanz war zunächst das CdS. Durch An- und Abklinguntersuchungen der Photoleitung im Bereich schwacher optischer Anregung wurde festgestellt, daß sich das Elektronenensemble allein beim Einsetzen einer solchen Störung in Bruchteilen einer Sekunde auf seinen neuen stationären Wert einstellt.

Erhöht man jedoch die Temperatur sehr rasch von kleinen Werten bis zur Meßtemperatur, so bleibt die Leitfähigkeit zeitlich träge hinter den zur jeweiligen Temperatur gehörenden stationären Werten zurück. Die Leitfähigkeit ist dabei um so träger, je geringer die erreichte Meßtemperatur ist (vergleiche Abb. 1) ${ }^{1}$. Der Zeitraum bis zum

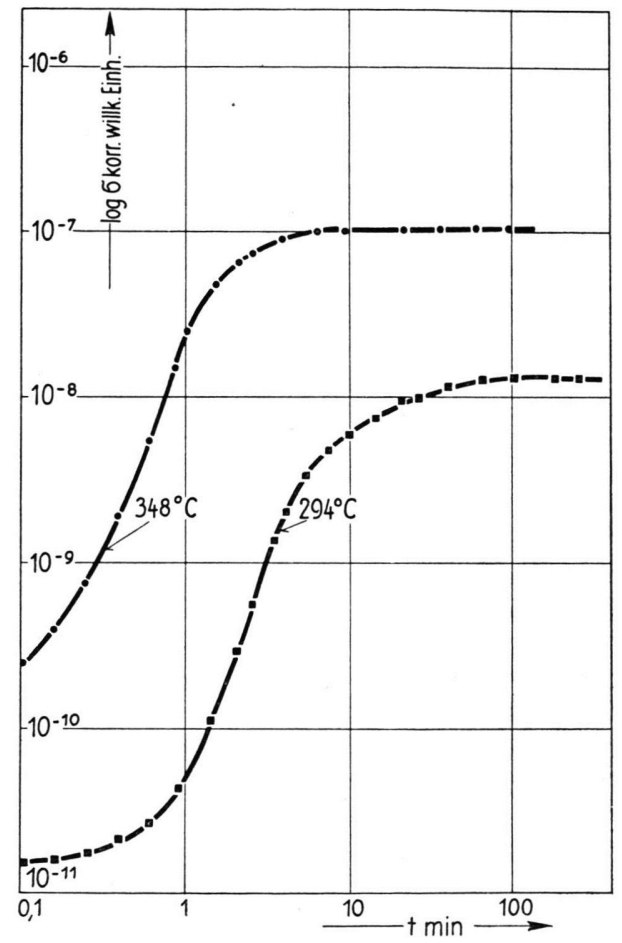

Abb. 1. Änderung der Leitfähigkeit eines CdS-Einkristalles bei „sprunghafter“ Änderung der Meßtemperatur von Zimmertemperatur auf den an den Kurven angegebenen jeweiligen Wert.

Erreichen eines stationären Wertes der Leitfähigkeit beträgt im untersuchten Falle bei $294^{\circ} \mathrm{C}$ etwa 50 und bei $348^{\circ} \mathrm{C}$ etwa $5 \mathrm{~min}$; er liegt also ganz beträchtlich oberhalb der Einstellzeit sowohl eines neuen Zustandes allein im Elektronenensemble als auch der Temperatur, die von $50^{\circ} \mathrm{C}$ bis zur Meßtemperatur in maximal 1 min anstieg. Es scheinen demnach für die Leitfähigkeit hier träge Effekte z. B. durch Lageänderung schwerer Gitterbausteine eine Rolle zu spielen.

In Abb. 2 ist eine Schar von Meßkurven der Leitfähigkeit

1. Verf. dankt Frau Dipl.-Phys. E. B o r c h a r d t für die freundliche Überlassung dieser Meßkurven. 
gezeichnet, die für verschiedene Aufheizgeschwindigkeiten derselben CdS-Probe erhalten wurden. Es ist deutlich, daß sich hierbei zwei Grenzkurven ergeben, eine tieferliegende für hohe Heizgeschwindigkeiten und eine darüberliegende für sehr kleine Heizgeschwindigkeiten.

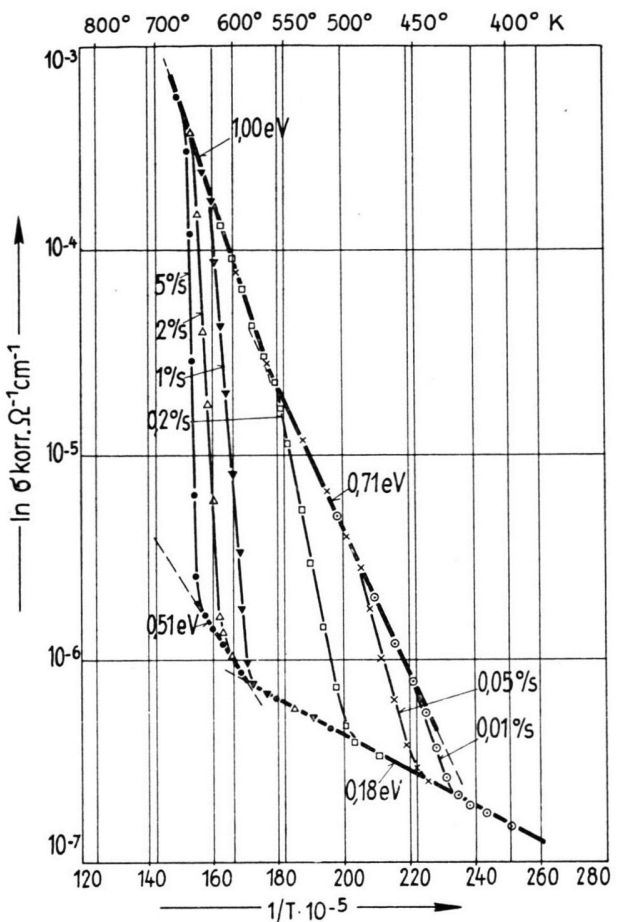

Abb. 2. Leitfähigkeit eines CdS-Einkristalles als Funktion der Temperatur; Scharparameter ist die Aufheizgeschwindigkeit.

Alle Meßkurven sind gut reproduzierbar, wenn man den Kristall nur langsam genug abkühlt ${ }^{2}$. Hat seine Leitfähigkeit jedoch irgend einen Wert der oberen Grenzkurve angenom-

2 Die Abkühlgeschwindigkeit muß kleiner als die geringste Aufheizgeschwindigkeit in Abb. 2 sein.

${ }^{3}$ In dem untersuchten Temperaturbereich folgen die Vor. gänge im Elektronenensemble der Temperaturänderung so rasch, daß sich für diese praktisch immer ein partielles Gleichgewicht einstellt. Versuchstechnisch lassen sich hier keine so großen Aufheizgeschwindigkeiten erreichen, daß das thermo- men und kühlt man den Kristall sehr schnell ab, so erhält man auch bei anschließendem schnellen Aufheizen bis zu jenem Leitfähigkeitswert lediglich die obere Grenzkurve.

Der Zustand des Kristalls ändert sich offensichtlich zeitlich träge mit der Temperatur und ist einfrierbar. Da im untersuchten Temperaturbereich oberhalb $250^{\circ} \mathrm{C}$ dieses Verhalten nicht mehr durch Fremddotierungen gestört wurde, liegt es nahe, zur Erklärung die thermodynamische Schaffung von Gitterfehlordnung heranzuziehen.

Gehen wir von dieser Annahme aus, dann läßt sich folgendes aussagen: Nur für sehr große Aufheizgeschwindigkeiten sollte es gelingen, die allein durch das Elektronenensemble bestimmte Leitfähigkeit an einem Kristall, dessen Fehlordnungsgrad eingefroren ist ${ }^{3}$, zu messen. Der exponentielle Leitfähigkeitsanstieg mit der Temperatur ist für die untere Grenzkurve einfach auszuwerten, zeigt jedoch, daß im Beispiel der Abb. 2 auch im Gebiet höherer Temperaturen wohl noch nicht von einer Eigenleitung gesprochen werden kann ${ }^{4}$.

Bei sehr geringen Aufheizgeschwindigkeiten verändert sich bei dem untersuchten Kristall bereits wenig oberhalb von $100^{\circ} \mathrm{C}$ der Fehlordnungsgrad mit der Temperatur innerhalb der Meßzeit. Man mißt zwar auch hier wieder eine elektronische Leitfähigkeit, die jedoch bei jedem Temperaturwert einem Kristall mit anderem Fehlordnungsgrad zugeordnet ist. Da der Fehlordnungsgrad sich exponentiell mit der Temperatur ändert, nimmt auch hier die Leitfähigkeit wieder exponentiell $\mathrm{zu}$, jedoch im allgemeinen um einen aus der Fehlordnungsenergie errechenbaren Wert vergrößerten Exponenten.

Damit wurde am Beispiel des CdS gezeigt, daß im beschriebenen Bereich höherer Temperaturen keine Eigenleitung vorliegt, mithin also die erste Gleichung nicht zur Deutung herangezogen werden darf. In diesem Gebiet tritt vermutlich eine Eigenstörstellenhalbleitung auf. Die rechnerische Auswertung der Meßergebnisse löst die einleitend beschriebene Diskrepanz bei der Bestimmung der Stromträgerbeweglichkeit.

Ähnliche experimentelle Ergebnisse, wie sie hier für das $\mathrm{CdS}$ angegeben wurden, sind beim $\mathrm{ZnO}, \mathrm{ZnS}$ und Se zu erhalten.

dynamische Gleichgewicht für das Elektronenensemble nicht erreicht wird. (Bei tiefen Temperaturen ist das jedoch durchaus möglich und für CdS-Kristalle hoher Dunkelleitfähigkeit unterhalb von Zimmertemperatur auch festgestellt worden.)

4 Die halbe Bandbreite liegt beim CdS in diesem Temperaturgebiet bei etwa $1 \mathrm{eV}$. 